\title{
Representation use and strategy choice in physics problem solving
}

\author{
Mieke De Cock* \\ Department of Physics and Astronomy and LESEC, KU Leuven, Celestijnenlaan 200C, box 2406, 3001 Heverlee, Belgium
} (Received 25 July 2011; revised manuscript received 25 July 2012; published 15 November 2012)

\begin{abstract}
In this paper, we examine student success on three variants of a test item given in different representational formats (verbal, pictorial, and graphical), with an isomorphic problem statement. We confirm results from recent papers where it is mentioned that physics students' problem-solving competence can vary with representational format and that solutions can be triggered by particular details of the representation. Previous studies are complemented with a fine grained analysis of solution strategies. We find that students use different problem-solving strategies, depending on the representational format in which the problem is stated.
\end{abstract}

DOI: 10.1103/PhysRevSTPER.8.020117

PACS numbers: $01.40 . \mathrm{Fk}$

\section{INTRODUCTION}

In recent literature on mathematics and physics education, a lot of attention is paid to student competence with different representational formats. By "representational format," we refer to the many different forms in which a particular concept or problem can be expressed and communicated, such as a graph, picture, free-body diagram, formula, etc. There is no purely abstract understanding of a physics concept-it is always represented in some form of representation. Therefore, being skilled in interpreting and using different representations and in coordinating multiple representations is highly valued in physics, both as a tool for understanding concepts and as a means to facilitate problem solving. Expert-novice studies have highlighted differences between expert and novice problem-solving approaches, with some of these differences involving representation use [1]. Expert physics problem solvers are fluent in their use of different representations, can easily translate between them, and can assess the usefulness of a particular representation in different situations.

Roughly, the skills needed in order to benefit from using external representations can be categorized in two groups.

(1) Representational fluency: Involves the ability to interpret or construct representations [2], as well as the ability to translate and switch between representations (on demand) accurately and quickly [3].

(2) Representational flexibility: Involves making appropriate representational choices in a given problemsolving or learning situation [4].

As it is a principal goal of most physics courses to produce adept problem solvers, a possible instructional aim might

\footnotetext{
*mieke.decock@fys.kuleuven.be
}

Published by the American Physical Society under the terms of the Creative Commons Attribution 3.0 License. Further distribution of this work must maintain attribution to the author(s) and the published article's title, journal citation, and DOI. be to develop this representational fluency and flexibility in physics students in order to benefit from using multiple representations.

Research concerned with representational issues has taken many approaches, in mathematics as well as physics, chemistry, and recently statistics education. A first aspect studied is student performance in particular representations. Student difficulties related to graphical representations have been studied in detail in both mathematics and physics education, where topics in kinematics received considerable attention [5]. Conceptual difficulties related to diagrammatic representations of electrical circuits have been addressed [6], as well as interpretation of ray diagrams in optics [7]. Also in chemistry, specific representational difficulties have been investigated [8]. In recent work on statistics education, Lem et al. [9] report on misinterpretations of histograms and box plots.

Besides student understanding of particular representations, use of multiple representations (MR) to learn concepts and problem solving has been studied in math and science education. In mathematics education, research evidence has shown that multiple representations can contribute to knowledge enhancement: the understanding that students gain by using MR is broader, deeper [10], more robust, and more flexible [11] than if students were to use single representations. However, despite the potential advantages of using MR in math problem solving and learning, an important number of studies $[2,10,12,13]$ should dissuade us from claiming that MR are beneficial in absolute terms. In order to benefit from using a representation, students should learn how to interpret the representation, how to connect it to reality, and how it relates to other representations of the same concept. Moreover, students need to be skilled at making choices among representations. If students do not have these prerequisites, MR can have a detrimental effect on their math problem solving and learning.

In science and physics education research (PER), several studies investigated potential benefits of the use of MR in 
courses for students' problem-solving ability [14]. Some studies compared student performance on problems that involve multiple representations to performance on problems that involve one single representation $[2,15]$ and some studies investigate student skill in translating between representations [16,17]. De Leone and Gire [18] studied how many representations students in a reformed course used when solving open-ended problems. Rosengrant et al. [19] investigated the use of free-body diagrams in solving problems in mechanics and static electricity. These studies show that students studying physics in an environment that puts emphasis on the use of MR are more inclined to construct multiple representations to solve problems themselves.

Besides being able to interpret specific representations and to relate different representations of the same concept, students also need the knowledge and skills to choose the most appropriate representation to solve a problem. In mathematics education, research on students' ability to make appropriate representational choices is not as common. Uesaka and Manalo [20] showed that a problem solver confronted with a multitude of representations is often unable to decide which representation(s) to choose, and for what purposes. Acevedo Nistal et al. [21] studied high school students' representational flexibility in linearfunction problems and found that the flexibility of a students' choice of representation can greatly affect his chance of succeeding in solving the problem at hand. In a later study [22], they also report on the justifications students' gave for their representational choices in linearfunction problems.

In PER, only a few papers [23-26] have broadened the study of representations to metarepresentational skills. Whereas the first three studies ask what students know about representations and how that knowledge might affect student performance, Kohl and Finkelstein [26] also investigated whether students can assess their own representational competence, their motives for handling a problem in a particular representation when given a choice, and whether providing this choice affects their performance.

The line of research in which the current study fits, focuses on the relationship between student success and the representational format in which the problem is posed: how does the representation in which the problem is formulated affect student performance and solution strategy?

Koedinger and Nathan [27] studied the performance and strategies of high school algebra students solving both word- and formula-based representations of problems and claim that there is a close link between solution strategies and representations, since certain representations elicit the use of certain strategies. Lem et al. [9] studied student performance in parallel distributional reasoning problems (statistics) formulated in terms of a histogram, box plot, or a list of descriptive statistics. Results show significant performance differences between representations, and the authors suggest a relation between misinterpretation and particular features of the different representations.

In Meltzer's work [28], students in an introductory algebra-based physics class are provided with quizzes that have nearly isomorphic problems formulated in four different ways (verbally, mathematically or numerically, graphically, and diagrammatically). This study also found instances where students performed significantly better in one representation of a problem than in another. Moreover, Meltzer found that students are not always consistent in their performance in a peculiar representation across topics.

In the work of Kohl and Finkelstein [26], student performance on problems in four different representational formats in the style of Meltzer (verbal, mathematical, graphical, and pictorial) is compared. Again, the authors found that there are statistically significant performance differences between different representations of nearly isomorphic statements of quiz and homework problems. When there was a performance difference between two formats, the mathematical format often was one of the formats involved. In that study, the mathematical format was the only format requiring an explicit calculation, whereas the other formats involved conceptual reasoning. Results show that, on average, students were more successful in the mathematical format, which is consistent with the idea that first-year students are more comfortable with "plug 'n chug" types of problems than with conceptual questions [29]. The examination was broadened by giving a group of students a choice of formats (before they read the problem), and by studying whether providing this choice affects their performance compared to students that were randomly assigned one of the four representational formats. It was found that allowing students to choose did not consistently increase or decrease success relative to the control groups, but that the effect varied strongly both across topic and across representation.

In this study, part of the work of Kohl and Finkelstein [26] is replicated and extended to gain more insight in representational fluency of physics students. Items developed by Kohl and Finkelstein were used as a starting point and student answers were studied and compared to the results mentioned by those authors. However, unlike Kohl and Finkelstein, we not only looked at the multiple choice answers, but we also asked the students for an explication and we extended the analysis by studying the written justifications in detail. These written explanations allow us to study the physical concepts and the problemsolving strategies that students use.

The main research questions thus are

(1) Do students perform differently when solving a physics problem formulated in different representational formats?

(2) How are the solution strategies of students affected by the representation used in the problem statement? 
Do students use different solution strategies in different representations?

\section{METHOD}

\section{A. Context and participants}

All three studies were conducted in a large-enrollment ( \pm 200 students) introductory physics class at the University of Leuven, Belgium. Students are typically first-year students, all taking the mandatory physics course as part of the pharmaceutical science program. Students' math skills are quite varied. The courses in the different studies are the first semester classes in the fall of 2009, 2010, and 2011. The format is mostly traditional, with some in-lecture concept tests and lecture demonstrations. Students have two two-hour lectures per week, and meet for three hours each week in a recitation session. Besides the lectures and the recitation sessions, there are three three-hour lab sessions.

The recitation sessions are generally traditional, with students discussing and solving (mostly traditional endof-chapter) problems with a graduate teaching assistant. Students' grades are based on exams, labs, and homework assignments. Homework problems consist mainly of qualitative conceptual problems (online).

\section{B. Data}

The sources of data for these studies are student responses to a selected midterm exam question: one research item was included in a midterm test. Participation in the test was free and test results did not count for the students' final grade. The representational format, in which the research item was formulated was randomly varied between students: one-third of the students solved the question formulated in the verbal format, one-third in the graphical, and one-third in the pictorial format. As a test item to study representational competence, we chose - in the first study - one of the questions developed by Kohl and Finkelstein [26]. More particularly, we translated the verbal, graphical, and pictorial version of the question on energy and energy transformation. The different versions of the item are shown in Fig. 1. In the follow-up studies, these items were slightly modified, based on the first findings. The rationale for the modification is discussed later; the modified questions are shown in Figs. 3 and 4.

As the main focus of the test was on conceptual understanding, it was decided not to use the mathematical format, which was in the original item explicitly asking to compute a (numerical) speed.

As mentioned in the work of Kohl and Finkelstein [26], these original problems are meant to be isomorphic from format to format, with the answers and distracters mapping from one format to the other. By isomorphic, the authors mean isomorphic from the point of view of a physicist.

\section{Method of analysis}

For all the test items, both the choices of the alternatives and the written explanations are studied in detail and results are reported.

First, the items are studied as pure multiple choice questions: only the alternative picked by the students, without considering the explanations, is taken into account. This means that also ticking the correct alternative with an erroneous reasoning or without an explication is counted as correct. In a second step, the explanations are also considered. An argument is then only evaluated as "correct" if it consisted of a correct physics reasoning and a correct conclusion was drawn. An incorrect physics reasoning or a correct argument but with a wrong conclusion was considered incorrect. Answers without justification were categorized as incorrect.

The results of this analysis in the different formats are compared and the argumentations of the students are studied in detail.

\section{RESULTS}

We start this section by presenting detailed results of the first study in which students solved one version of the original item developed by Kohl and Finkelstein [26]. In the second part, results of a second and third study are discussed. These studies are based on modifications in the original item. The presented tables contain data of all three studies, for reasons of compactness.

\section{A. Initial study: Searching for representational effects}

In the first study, students solved one of the items shown in Fig. 1. In this section, we focus on comparisons of student performances on this original test problem in different representational formats and on the solution strategy students chose in the different formats.

\section{Performance in different representational formats}

In Table I, the fraction of students that answered the test item correctly is shown. $N$ represents the number of students answering the question in the different formats.

All statistical significance tests involving student success rates are two-tailed binomial $z$ tests. We consider a difference with $p>0.10$ as not significant, $p$ between 0.10 and 0.05 marginally significant, $p$ between 0.05 and 0.01 significant, and $p<0.01$ highly significant.

When considering only the answer options $(a-b-c-d)$ and not the explanations ("correct alternative" in Table I), the difference between the verbal and graphical format is marginally significant $(p=0.07)$, whereas the other differences are not significant. This is in line with the results in Kohl and Finkelstein [26]

However, when taking into account the explanations given by the students, results look quite different. Table I 


\section{Verbal format}

A professor drops a ball from the top of an eight-story physics building.

At what point has the ball reached half of the speed it has just before it hits the ground? Neglect air resistance.

A. The ball has reached half of its final speed when it has fallen two stories.

B. The ball has reached half of its final speed when it has fallen four stories.

C. The ball has reached half of its final speed when it has fallen six stories.

D. The ball has reached half of its final speed at some other point.

Explain.

\section{Graphical format}

A roller coaster car approaches a hill going just fast enough to reach the top and stop.

A graph of the hill's height versus its horizontal coordinate is shown.

At what point has the car slowed down to half of its original speed? Neglect friction.

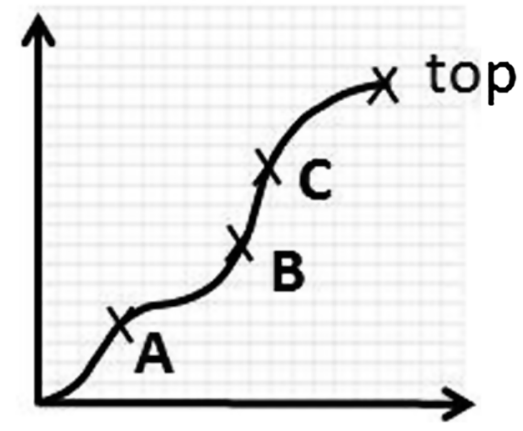

A. Point $A$

B. Point B

C. Point $\mathrm{C}$

D. Somewhere else

Explain.

\section{Pictorial format}

A roller coaster car comes to rest at the top of a hill before starting down the other side. At what point on the track is the car moving at half of the speed it has at the bottom of the hill? Ignore friction.

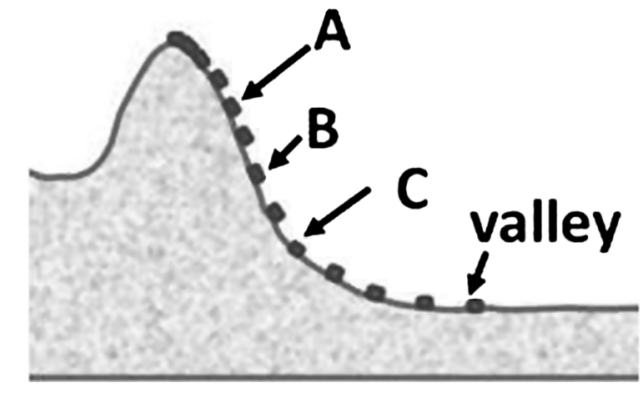
A. Point A
B. Point B
C. Point C
D. Somewhere else

Explain.

FIG. 1. Verbal, graphical, and pictorial representation of original item [26].

also shows the fraction of students choosing the right alternative and giving a correct explanation for their choice.

When comparing these results, we find that the differences between verbal and graphical format and between verbal and pictorial format are highly significant ( $p=0.0006$ and $p=0.0005$, respectively), whereas graphical and pictorial format do not differ significantly from each other. It is almost immediately clear that the fractions of correct answers with or without taking into account the explications do not differ significantly for the verbal format $(p=0.72)$, but the difference is highly significant for the pictorial $(p=0.006)$ and the graphical format $(p=0.003)$. 
TABLE I. Fraction of students answering correctly, broken down by representational format, for the different problem variants.

\begin{tabular}{lccc}
\hline \hline & Verbal & Graphical & Pictorial \\
\hline Study I & $N=62$ & $N=64$ & $N=63$ \\
Correct alternative & 0.44 & 0.28 & 0.33 \\
Correct alternative + correct justification & 0.40 & 0.08 & 0.13 \\
Study II & $N=53$ & $N=65$ & $N=63$ \\
Correct alternative & 0.45 & 0.23 & 0.30 \\
Correct alternative + correct justification & 0.13 & 0.08 & 0.16 \\
Study III & $N=59$ & $N=57$ & $N=54$ \\
Correct alternative & 0.44 & 0.53 & 0.28 \\
Correct alternative + correct justification & 0.31 & 0.25 & 0.20 \\
\hline \hline
\end{tabular}

\section{Solution strategy in different representational formats}

As we did not expect these results, we looked in detail at the students' explanations in order to find out how these differences can be explained. We found that students justify their answers differently in different representational formats. Some explanations use kinematics, some use conservation of energy or are based on a linear reasoning argument. There also were arguments which are strongly tied to the visual information in the provided representation.

In what follows, different solution strategies are discussed.

Conservation of energy versus kinematics.-In Table II, the fractions of answers using kinematics and conservation of energy (not necessarily in a correct way) are shown for the different formats. The number of students answering the question is indicated in parentheses.

The difference between fractions of answers using kinematics in the verbal and graphical format is highly significant ( $p=0.00006)$, as is the difference between the verbal and pictorial format $(p=0.00006)$. The difference between the graphical and pictorial format is not significant.

When comparing the fractions of answers based on conservation of energy, the difference between the verbal and pictorial format turns out to be highly significant ( $p=$ $0.0017)$; the difference between the verbal and graphical format is significant $(p=0.042)$, while the difference between the graphical and pictorial format is not significant.

When looking within the different formats, it turns out that the fraction of students using a kinematics argument differs significantly from the fraction of students writing an explanation based on conservation of energy for the verbal $(p=0.00006)$ and pictorial format $(p=0.00006)$. However, for the graphical format, the difference between the fractions is only marginally significant $(p=0.053)$. In the verbal format, more students solved the problem using kinematics than using energy conservation, whereas for both other formats, more justifications are based on energy conservation. Out of the 25 students that solved the item correctly in the verbal representation, 19 of them justified their answer using kinematics, while only 5 students based their argumentation on conservation of energy. One student used a correct combination of energy conservation and kinematics to explain his answer. In the graphical and pictorial format, none of the explications based on kinematics is correct. The correct explanations in these formats are all based on conservation of energy.

These results confirm that the different formats are not completely isomorphic: the verbal format is one dimensional and as such leaves the possibility to solve the problem using kinematics. This is not the case for the other two formats, where the two-dimensional character prevents the easy use of kinematics. More interestingly, the results also suggest that, if possible, students are more likely to apply kinematics than to apply conservation of energy. However, the data for the graphical and pictorial format seem to suggest that students are aware of the difficulty of the use of kinematics in these situations: a smaller fraction of students uses a kinematics argument in these situations than in the verbal formulation.

Conservation of energy.-In a next step, we studied the "conservation of energy answers" in detail. We found that

TABLE II. Fraction of answers with justification based on kinematics and conservation of energy in different representational formats.

\begin{tabular}{lcccccc}
\hline \hline & & Kinematics & \multicolumn{3}{c}{ Energy conservation } \\
& Verbal & Graphical & Pictorial & Verbal & Graphical & Pictorial \\
\hline Study I & $0.56(62)$ & $0.22(64)$ & $0.13(63)$ & $0.21(62)$ & $0.38(64)$ & $0.48(63)$ \\
Study II & $0.28(53)$ & $0.17(65)$ & $0.30(63)$ & $0.49(53)$ & $0.29(65)$ & $0.40(63)$ \\
Study III & $0.19(59)$ & $0.14(57)$ & $0.09(54)$ & $0.66(59)$ & $0.56(57)$ & $0.78(54)$ \\
\hline \hline
\end{tabular}


a lot of students have trouble applying the principle of conservation of energy correctly. In Table III, we present the number of students using a correct energy conservation explication. The number in parentheses indicates the number of students using an energy argument in that format.

Analysis of the errors shows that we can distinguish between two groups of errors. The first error that is frequently seen is a wrong interpretation of

$$
E_{\text {mech }}=E_{\text {kin }}+E_{\text {pot }}=\text { const. }
$$

A lot of students write, e.g., in the graphical format, correctly, $E_{\text {pot }}^{f}=E_{\text {kin }}^{i}$, but then wrongly generalize this expression to $E_{\mathrm{pot}}=E_{\mathrm{kin}}$. Using this equation in the form

$$
m g h_{f}=m \frac{v_{i}^{2}}{2}
$$

they then conclude that, "if $v=v_{i} / 2$, then $h=h_{f} / 4$, so half of the final speed is reached after the car has surmounted one-fourth of the total height."

A second class of errors is related to students misinterpreting their own notation. Again, these students write, in the verbal format, e.g., correctly, $E_{\mathrm{pot}}^{i}=E_{\mathrm{kin}}^{f}$, leading them to state that

$$
m g h_{i}=m \frac{v_{f}^{2}}{2},
$$

or $v_{f}=\sqrt{2 g h_{i}}$. From this expression, they conclude that, "if $v=v_{f} / 2$, then $h=h_{i} / 4$, and thus half of the final speed is reached at a height 4 times smaller than the total height." These students incorrectly interpret the " $h_{i} / 4$ " as the point at height $h_{i} / 4$ instead of the correct interpretation, i.e., the ball reaches a velocity $v_{f} / 2$ after it has fallen $\Delta h=h_{i} / 4$.

Linear reasoning.-An interesting distracter is answer $b$ in each of the formats. This alternative could correspond to a "linear reasoning": "half of the final speed is reached halfway." In mathematics education, a vast amount of research has shown that students have a strong tendency to apply linear or proportional models anywhere, even in situations where they are not applicable [30,31].

In Table IV, the fraction of linear answers (i.e., the fraction of students answering alternative $b$ ) is given for the different formats.

It turns out that the difference between verbal and graphical format is highly significant $(p=0.00002)$ and

TABLE III. Distribution of answers using correct argument based on energy conservation.

\begin{tabular}{lccc}
\hline \hline & Verbal & Graphical & Pictorial \\
\hline Study I & $0.38(13)$ & $0.21(24)$ & $0.27(30)$ \\
Study II & $0.23(26)$ & $0.26(19)$ & $0.28(25)$ \\
Study III & $0.46(39)$ & $0.44(32)$ & $0.26(42)$ \\
\hline \hline
\end{tabular}

between verbal and pictorial format is significant ( $p=$ 0.012 ). The difference between graphical and pictorial format is not significant $(p=0.2)$.

When analyzing the explanations of students picking alternative $b$, it becomes clear that not all of them refer to a "natural" linear reasoning: only 5 out of 27 students in the graphical and 1 out of 20 students in the pictorial format simply state that "the car will have half its final speed when travelled $h / 2$ " as argumentation. Most of the students do argue their choice for the linear alternative in more detail. We can distinguish between two main errors. The first reasoning can be summarized by the following:

$$
\begin{aligned}
& \text { As } v=a t, v \text { changes linearly, so it will reach half its } \\
& \text { maximal value at half height. }
\end{aligned}
$$

The second error which often shows up can be stated like (for the pictorial problem)

Initially, there is only potential energy. This is transformed in kinetic energy while moving down. When the height is half the original height, the potential energy is reduced to half its initial value, which means that the kinetic energy has increased to half its final value. Therefore, at $h / 2$ the car travels with half the final velocity.

Representation-dependent cueing.-From the above, it is clear that both performance and physics principles used by students differ between representational formats. Conservation of energy, kinematics, and linear reasoning are already mentioned, but another class of arguments seems to be strongly related to the representational format: quite a few students explain their choice by referring to the picture or graph in the question. Some of these students are mistaking the picture of the hill or the vertical direction (horizontal direction) graph for the position (time) graph and refer to the slope or tangent as the velocity. Two examples are shown in Fig. 2. Seven students (11\%) make this mistake in the pictorial format, $10(16 \%)$ in the graphical, but, of course, none in the verbal version. Some students refer to the graph or picture in their argumentation in a very incoherent way. We consider these arguments as examples of representation-dependent cueing, where subtle features of the problem representation have a significant impact on student success [32].

TABLE IV. Fraction of students choosing the linear alternative in the different representational formats.

\begin{tabular}{lccc}
\hline \hline & Verbal format & Graphical format & Pictorial format \\
\hline Study I & $0.13(62)$ & $0.42(64)$ & $0.32(63)$ \\
Study II & $0.11(53)$ & $0.32(65)$ & $0.27(63)$ \\
Study III & $0.15(59)$ & $0.23(57)$ & $0.30(54)$ \\
\hline \hline
\end{tabular}



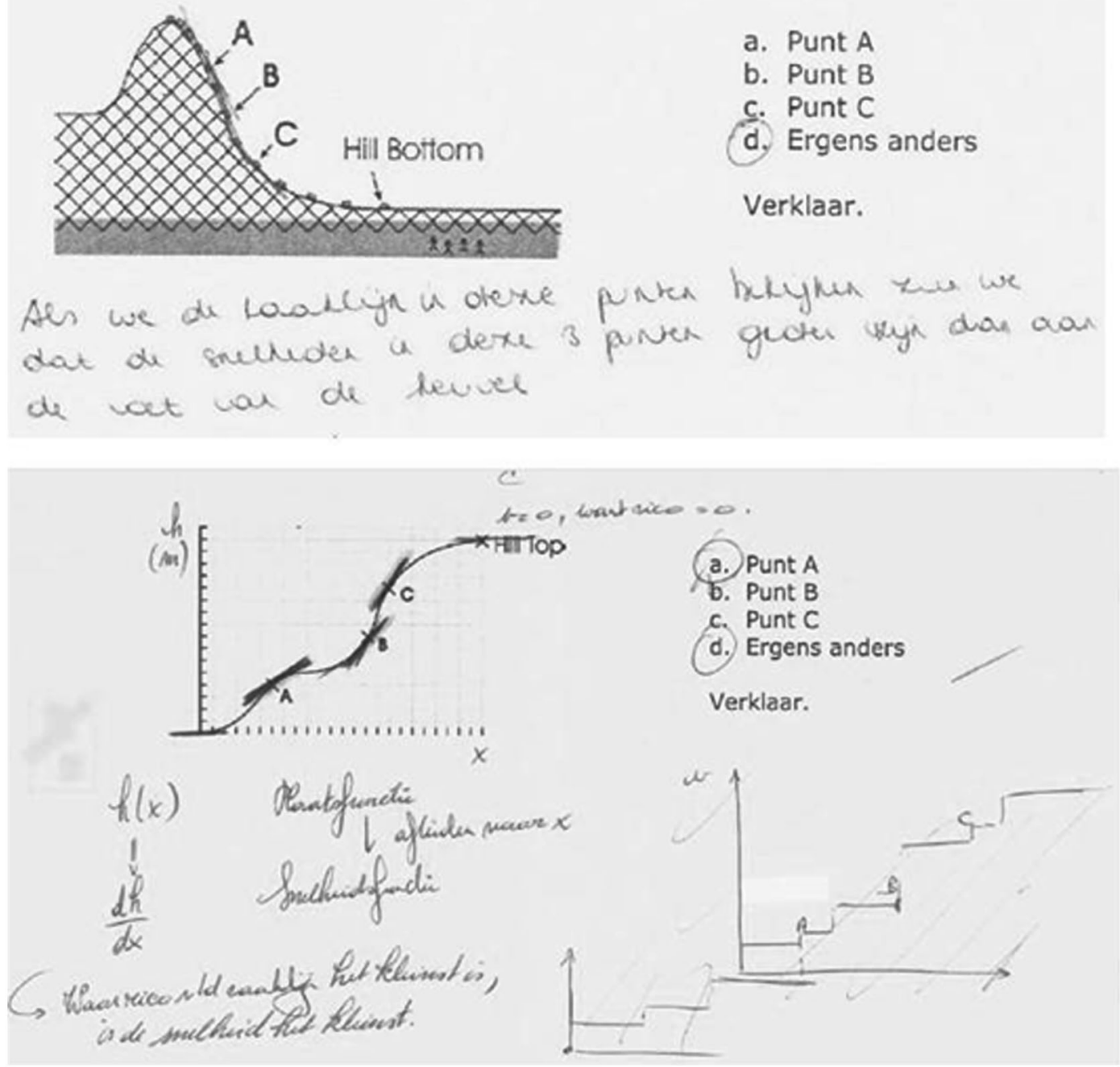

FIG. 2. Examples of student answers confusing the slope of the picture or graph with the velocity.

\section{B. Follow-up studies}

The detailed analysis of the student answers in the initial study revealed that the differences in accuracies between the representational formats might be explained by the fact that not all problems can be solved by using the same physics ideas. As mentioned, the verbal format can be solved by using conservation of energy or by using 1D kinematics while this is not the case for the graphical and pictorial version of the problem. This raises the question of whether the differences are due to the representation or not. We therefore adopted the test items in such a way that they are more alike from the point of view of possible physics ideas to solve them.

In a first follow-up study, the test items were reformulated in such a way that they all can be solved either by $1 \mathrm{D}$ kinematics or by conservation of energy. The exact formulation is shown in Fig. 3. In the second follow-up study, the problems again are modified. As can be seen in Fig. 4, they now can be solved only by using a conservation of energy argument.
Again, we report on performances in the different representational formats and we discuss student solution strategies.

Concerning the performance differences between the formats, we expect, based on the analysis of the initial study, these to reduce when the problem statements are more equivalent. For the solution strategies, we expect that the fraction of student arguments based on kinematics will increase in the second variant but reduce in the third version, where we think more students will justify their answers based on energy conservation. Together with an increase in kinematics arguments, we also predict more correct explanations in the second variant, whereas an increase in energy justifications might bring along a reduction in correct answers for the third variant.

\section{Performance in different representational formats}

Table I shows the fractions of students answering the test item correctly, both with and without the explanation taken into account, for all three versions of the 
A lift is tilted against a wall. At the eight floor, the cable breaks down and a box starts sliding downwards along the lift.

At what point has the box reached half of the speed it has just before it hits the ground? Ignore friction.

A. The box reaches half of its final speed when it has fallen two stories.

B. The box reaches half of its final speed when it has fallen four stories.

C. The box reaches half of its final speed when it has fallen six stories.

D. The box reaches half of its final speed at some other point.

Explain.

While moving a box with a lift, the cable breaks down and the box starts sliding downwards.

At what point has the box reached half of the speed it has just before it hits the ground? Ignore friction.

$\begin{array}{ll}\text { A. } & \text { Point A } \\ \text { B. Point B } \\ \text { C. Point C } \\ \text { D. Somewhere else } \\ \text { Explain. }\end{array}$

While moving a box with a lift, the cable breaks down and the box starts sliding downwards.

Agraph of the height of the box as a function of its horizontal position is shown.

At what point has the box reached half of the speed it has just before it hits the ground? Ignore friction.
A. Point A
B. Point B
C. Point $\mathrm{C}$
D. Somewhere else

Explain.

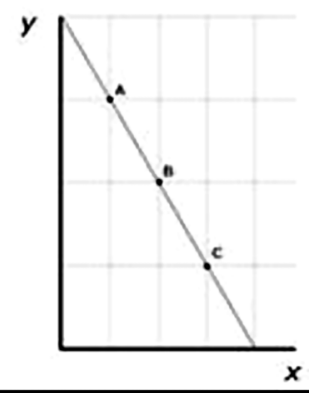

FIG. 3. Verbal, pictorial, and graphical representation of second study.

problem. The results are broken down by representational format.

When considering only the answer option and not the explanation, in none of the cases the differences are highly significant, except for the difference between graphical and pictorial format in the third study. When taking into account student explanations, we found that the results for the verbal format differed significantly from the other two formats in the initial study. In the follow-up studies, we do see these differences disappear, as we expect: the fractions of correct answers with a correct justification are not statistically different between the formats in studies II and III. However, the number of correct justifications is not in line with our expectations: the number of correct justifications is not increasing in the second study, while it is for the graphical format in the third one.

\section{Solution strategies}

To understand why the pattern in justification accuracies was not as expected, we again looked in detail at the student responses.

Conservation of energy versus kinematics. - In the initial study, we saw that students were more inclined to apply 1D kinematics than energy conservation in situations where this was possible (verbal format). The test items were therefore modified in such a way that all formats leave 
As John is always late, his father installed a slide. The wavy slide starts from the window of John's room at the fourth floor. Every morning, John is happy to slide down four stories.

At what point does John reach half of the speed he has just before he hits the ground? Ignore friction.

A. John reaches half of its final speed when he has slide down one story.

B. John reaches half of its final speed when he has slide down two stories.

C. John reaches half of its final speed when he has slide down three stories.

D. John reaches half of its final speed at some other point.

Explain.

As John is always late, his father installed a slide. The wavy slide starts from the window of John's room. Every morning, John is happy to slide down.

At what point does John reach half of the speed he has just before he hits the ground? Ignore friction.

A. Point A

B. Point $B$

C. Point C

D. Somewhere else

Explain.

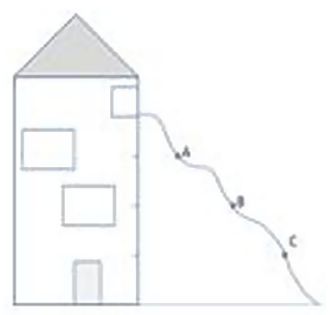

As John is always late, his father installed a slide. The wavy slide starts from the window of John's room. Every morning, John is happy to slide down.

A graph of the height of the slide as a function of its horizontal position is shown.

At what point does John reach half of the speed he has just before he hits the ground? Ignore friction.

$\begin{array}{ll}\text { A. } & \text { Point A } \\ \text { B. } & \text { Point B } \\ \text { C. } & \text { Point C } \\ \text { D. } & \text { Somewhere else } \\ \text { Explain. } & \end{array}$

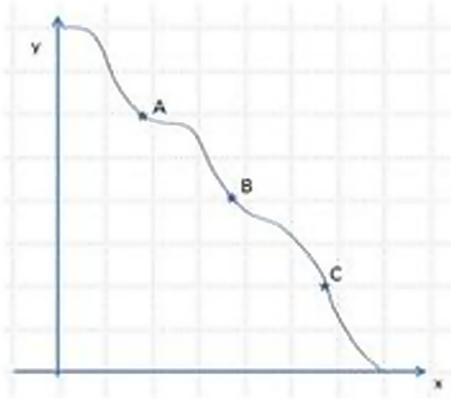

FIG. 4 (color online). Verbal, graphical, and pictorial representation of third study.

the possibility to solve it by using both 1D kinematics and conservation of energy.

In Table II, the fractions of justifications based on either of these possibilities are shown.

The results are not in line with our hypothesis: the fraction of kinematics arguments is not increasing in study II, except for the pictorial format. For the verbal format, the decrease is highly significant; for the pictorial format, the increase is significant; there is no difference for the graphical format.
The number of arguments based on energy conservation is increasing for the verbal format $(p=0.0016)$ but is not different for the graphical and pictorial format.

In a second modification, the items were adapted such that they have to be solved using energy conservation. We do see the expected changes in the solution strategies, from study II to III: the number of kinematics arguments is decreasing or remains the same, but the number of energy arguments is increasing. This increase is highly significant for both the graphical and pictorial format. 
As the graphical and pictorial formulations of the item in the original and third study are very similar, we expected no difference between these results. We cannot explain the (highly) significant differences between the fractions based on energy conservation, although we have a hypothesis related to representation-dependent cueing. This is discussed later.

Energy conservation.-The same errors that were discussed for the initial study show up in studies II and III. The number of students using correct energy argumentations is given in Table III, but the numbers are too small to draw any statistical conclusion.

Kinematics.-As discussed, we expected the number of kinematics arguments to increase in the second study, but this was not the case. Moreover, the few students that tried to solve the problem using kinematics did not do it properly. Most of them considered the motion as one dimensional but did not indicate a clear choice for an axis. They did not take into account in the calculation that the motion is not vertical and simply took $g$ for the acceleration.

Linear reasoning.-We expected the number of linear answers to increase in the second study, this being cued by the line representing the path of the box, but this turned out not to be the case. The reasoning errors made to end up with answer $b$ are the same as in the initial study.

Representation-dependent cueing.-In the follow-up studies, we again found some instances of representationdependent cueing. In the graphical condition of the second study, two students interpreted the graph as an $x(t)$ graph, concluding that the velocity (or slope) is constant. Another student argued that the velocity is the area under the curve, and that neither for $A, B$, nor $C$ this area is half the total area. One student took the points $A, B, C$ in the $y(x)$ graph

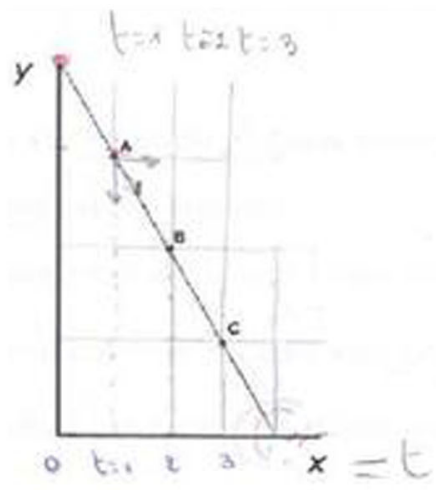

a. De snelheid van kast en platform is de helft van de eindsnelheid in punt $A$.

(b. De snelheid van kast en platform is de helft van de eindsnelheid in punt 8.

c. De snelheld van kast en platform is de helft van de eindsnelheid in punt $\mathrm{C}$.

d. De snelheid van kast en platform is de helft van de eindsnelheid op een ander punt.

Verkiaar.
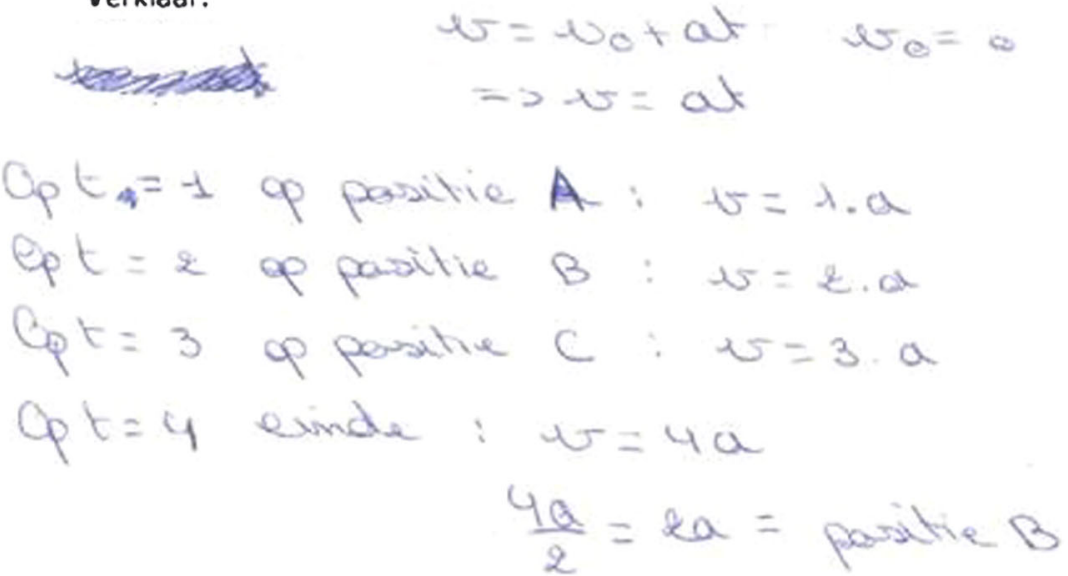

FIG. 5 (color online). Student answer mistaking the $y(x)$ graph for a $y(t)$ graph. 
of the second study as the place of the cupboard on different moments in time. The student answer is shown in Fig. 5.

Also in the graphical version of the third study, we found examples where students referred to the slope of the curve: "for the velocity to be half of the final velocity, the slope should be half of the slope at the end." In the pictorial version of the third study, we did not find any reference to the slope, while we did in the pictorial version of the initial study.

\section{Multiple representations}

The students in these studies were provided with single representations in the problem formulation, but many of the students' papers showed that they used supplementary representations to solve the problem: a sketch or a drawing, a graph, equations, numerical values, etc.

Although in none of the problem formulations equations were provided, an overwhelming number of students (415 out of 540, i.e., 77\%) wrote down an equation(s) in their argumentation, sometimes together with a sketch or a graph. The use of equations was most prominent in the verbal format in all three studies $(85 \%, 65 \%$, and $61 \%$ in the verbal, pictorial, and graphical format, respectively, in study I, $91 \%$ versus $73 \%$ and $69 \%$ in the second study, and $88 \%$ versus $80 \%$ and $77 \%$ for the last study). In both the first and the second study, the fraction in the verbal format differs significantly from the fractions in the other formats at the $p=0.01$ level. This is not the case in the third study.

Besides equations, a sketch or picture was often added by the students to support their explanation. Most often, this consisted of a simple drawing of the situation, and sometimes (but certainly not always) also included an axis. This almost exclusively showed up in the answers on the problems formulated in the verbal format ( $48 \%$ in study I, $75 \%$ in study II, $61 \%$ in study III).

Twelve answers (out of 540) included an extra graph. This was always an $x(t), v(t)$, or $a(t)$ graph.

A last remarkable aspect related to the use of multiple representations is the use of concrete numerical values. A substantial number of students plugged in concrete numbers, e.g., by writing "Suppose the building is $16 \mathrm{~m}$ ..." or by putting numbers on the picture or graph. In this way, they turned a conceptual question into a quantitative one. From all students (540) in the three studies together, 159 (29\%) of them used this strategy. Breaking it down by format, $66 \%, 20 \%$, and $8 \%$ of the students plugged in numbers for the verbal, graphical, and pictorial format in the first study, $55 \%, 15 \%$, and $14 \%$ in the second study, and $36 \%, 26 \%$, and $26 \%$ in the third study. In the first and second study, the fraction in the verbal format differs significantly from the fractions in the other formats at the $p=0.001$ level; this is not the case in the third study.

\section{DISCUSSION AND CONCLUSION}

In these studies, we wanted to investigate the role of representational format in student performance when solving physics problems. The work of Meltzer [28] and Kohl and Finkelstein [26] indicates that student performance can vary strongly with the representation used in the formulation of a question. By asking students for detailed explanations, we wanted to gain more insight in these differences. Kohl and Finkelstein [26] suggest that performance differences depend on a number of things, including student expectations, prior knowledge, metacognitive skills, and the specific contextual features of the problem and the representations. Moreover, they argue that different problem representations might prompt different solution strategies.

We conducted three studies; in each of them a variant of one test item was included. As we asked students to explain their choice, we could study the student answers both as a function of representation and as a function of possible physics concepts in the solution.

Detailed study of the student explanations revealed that the differences in the first study between the different representational formats in the problem formulation can - to a large extent-be explained by the fact that students use solution strategies based on different physics ideas to explain their answers in the different formats. The problem formulation of the verbal format allows the use of both 1D kinematics and conservation of energy to solve it. This is not the case in the graphical and pictorial formats, where students should use conservation of energy to argue their choice. Detailed analysis of the data does confirm that students use the 1D kinematics often in the verbal format and that most of the correct answers are based on a kinematics argument. This is in line with results presented in Walsh et al. [33], where a similar problem is offered to students and where it is also reported that (in their case) all students solved the problem using kinematics. The number of correct explications based on conservation of energy is surprisingly small in all three formats.

This raises the question of "isomorphism of questions." As mentioned in the Introduction, the problems were meant to be isomorphic "from the point of view from an (expert) physicist," but in the original article [26], there is no further specification on the interpretation of this statement. In Simon and Hayes [34], problems are defined to be isomorphic if they can be mapped to each other in a one-toone relation in terms of their problem-solving trajectories. Singh [35] defines isomorphic problems as problems that require the same physics principle(s) to solve them.

Although it seems reasonable to state that the problems are isomorphic in the sense of "being the same from the point of view of an expert physicist," it is not as evident that they are isomorphic in the sense of Simon and Hayes or Singh: the verbal format can be solved using two different solution trajectories, while this is not the case for the 
pictorial and graphical format. In this sense, there is no one-to-one mapping between the solutions of the verbal problem and the other two.

One could therefore argue that, in this first study, it is unclear whether the differences are due to the different representations or whether results are different because the problems are not completely parallel. In the follow-up studies, questions were reformulated in order to make them more parallel in the sense of the definition of Simon and Hayes [34]. Again, student arguments were studied in detail.

By reformulating the item in such a way that all formats allowed a solution based both on 1D kinematics and on energy conservation (study II), we could study in more detail students' choices for a particular solution strategy. From the results in the verbal format in the first study, we expected the number of student arguments based on kinematics to increase in the second study. Except for the pictorial format, this turned out not to be the case. Moreover, whereas in the verbal format of the initial study, using (1D) kinematics was a successful strategy, this was not the case in the second study. The reason for this is probably related to the fact that the initial problem was simply a vertical free fall situation, whereas in the modified version, the sliding of the cupboard was not vertical but an angle had to be taken into account. Most of the students did not take into account this angle in their argumentation; nevertheless, they did end up with the correct alternative. One could argue whether this is also an example where a small change in representation (vertical versus tilted) has an impact on student success.

In the third study, the items were adopted in such a way that all formats have to be solved using the same (single) physics principle (energy conservation), so the "only" variable is the representational format. Our results show that performance differences disappear and that problemsolving strategies are very similar in the different formats. This is not really in line with former results in the literature where it is mentioned that student performance can sometimes (strongly) vary with representational format. We cannot explain the increase in correct answers in the third study.

An analysis of the alternative answers also shows that the fraction of students choosing the linear alternative is larger in the graphical and pictorial questions. In mathematics education, it is well known that students show a tendency to overrely on a linear model [30]. A possible reason for the difference between the frequencies of the linear answer might be found in this tendency. It looks like in the graphical and pictorial format of the first study, more students experience difficulty solving the problem using physics arguments and this might be the reason why they "fall back" to the "intuitive linear reasoning." The research in mathematics education shows that students have great difficulties in explaining why their methods are correct. Interview data indicate that students typically use the linear model in a spontaneous way and do not check whether the model is applicable in a given situation. They do not seem to have clear arguments justifying its use, nor do they realize that there are other, competing, models. To evaluate whether this argument holds true, justifications of students choosing the "linear answer" were studied in detail. It turned out that "intuitive linear reasoning" is not the main argument used. This somehow is in line with results in De Bock et al. [31] where a more ambivalent picture of students' overuse of linearity in physics shows up: although linear reasoning is sometimes used as a default strategy, this study also indicates that in physics the context is more taken into account than is suggested by research on math problem solving. However, why there are more linear answers in the graphical and pictorial format still remains unclear.

In all three studies we found evidence consistent with the idea that specific, microlevel features of a representation can prompt students to a particular solution strategy or argument, as is suggested in Kohl and Finkelstein [26]. In both the graphical and pictorial formats, instances of student arguments based on specific features in the graph or picture were found. Of course, we did not find such examples in the verbal formats. However, in the pictorial version of the third study, we did not find any reference to the slope of the "graph" while we did so in the pictorial version of the first study. One hypothesis to explain this could be the fact that the picture in the initial item more strongly suggests the interpretation as a graph than does the picture with the house. This also might be a reason for the higher number of energy conservation arguments in the pictorial version of the third study compared to the first. If this hypothesis is valid, it can be considered as another example of representation-dependent cueing.

Concerning the use of multiple representations, it was found that a lot of students used additional representations while solving the problem. In an overwhelming number of student answers, this additional representation was a (set of) equation(s). This is not surprising. It is well known in literature that students show a strong tendency to solve problems by "plugging and chugging," in a more or a less structured way. A second remarkable aspect involves the use of numerical values in the problem solution, while these are not given in the problem statement. By doing so, students turned a qualitative question into a quantitative one, allowing them to use equations and calculate concrete numbers. Work done by Singh [35] suggests that this might be a smart strategy as she found that students solving coupled quantitative and conceptual questions can leverage their quantitative solution to correctly answer the corresponding conceptual questions. However, Singh [35] also reports that, almost without exception, students avoided turning conceptual questions into quantitative ones, even when explicitly encouraged to do so. In our data, we do 
find a lot of examples where students spontaneously apply this strategy. However, less than half of the students that adopted this strategy were successful. Only in the verbal format of the first study, 50\% of the students that plugged in concrete numbers ended up with the correct answer.

In summary, we found that student performance can be affected by the representational format of the problem statement. We found instances of student answers being clearly triggered by some specific aspects of the provided representation. Moreover, our data suggest that students' use of additional representations is related to the representation used in the problem formulation. Finally, detailed analysis of student answers showed that, in the studied problem, student success to a large extent depends on the physics concept to be used in the solution.

The current study has a number of limitations. First, only one problem in three representations was studied. This makes it difficult to formulate general conclusions on student representational fluency. On the other hand, the different variations in the problem formulation and the in-depth analysis of the student answers gave us insight into the subtle details that can play a role in student success on a particular problem. Second, the graphical and pictorial format of the problem are quite similar. It might be interesting to include representations that are also visually more different. Third, students were provided with a particular representation and had no choice. In future research, we will study students' representational adaptivity in more detail. By representational adaptivity, we refer to students' ability to make appropriate representational choices, i.e., choices that match task demands and also take the characteristics of the subject that has to make the choice and the context into account [36]. We will set up a study where students can chose a representation once they have read the problem statement.

Although developing or testing new instructional material was not a goal of the presented research, we can think of implications for instruction of our results. We again found examples of small or subtle representation characteristics affecting student solutions. Instructors should be aware of this sensitivity to representations, especially when student performance is evaluated in one single representation: a student might be able to apply a concept in a familiar context using a certain representation but fail when the representation is changed. The careful analysis of concepts used in students' answers made us aware of the subtlety of "isomorphism from a physicist's point of view." What instructors consider "the same problem" might be perceived differently by the students.

Besides being able to decode, interpret, and derive information from a given representation, students also should learn how to choose appropriate representations to solve a given problem. Much more research effort needs to be invested to determine what can be considered a flexible representational choice and to design powerful learning environments explicitly addressing representational flexibility.

\section{ACKNOWLEDGMENTS}

This research was partially supported by an "Impulsproject Vakdidactisch Onderzoek" of the KU Leuven.
[1] J. Larkin, The role of problem representation in physics, in Mental Models, edited by D. Gentner and A. Stevens (Lawrence Erlbaum, Mahweh, NJ, 1983); M. T. H. Chi, P. J. Feltovich, and R. Glaser, Categorization and representation of physics problems by experts and novices, Cogn. Sci. 5, 121 (1981); L. Hsu, E. Brewe, T. M. Foster, and K.A. Harper, Resource letter RPS-1: Research in problem solving, Am. J. Phys. 72, 1147 (2004).

[2] S. Ainsworth, P. Bibby, and D. Wood, Analyzing the costs and benefits of multiple-representational learning environments, in Learning with Multiple Representations, edited by M. W. Someren et al. (Pergamon, New York, 1998).

[3] R. Even, Factors involved in linking representations of functions, J. Math. Behav. 17, 105 (1998).

[4] A. Acevedo Nistal, W. Van Dooren, G. Clarebout, J. Elen, and L. Verschaffel, Conceptualising, investigating and stimulating representational flexibility in mathematical problem solving and learning: A critical review, ZDM Math. Educ. 41, 627 (2009).
[5] R. J. Beichner, Testing student interpretation of kinematics graphs, Am. J. Phys. 62, 750 (1994); L. McDermott, M. Rosenquist, and E. van Zee, Student difficulties in connecting graphs and physics: Examples from kinematics, ibid. 55, 503 (1987); F. M. Goldberg and J. H. Anderson, Student difficulties with graphical representations of negative values of velocity, Phys. Teach. 27, 254 (1989).

[6] S. Johsua, Students' interpretation of simple electrical diagrams, Eur. J. Sci. Educ. 6, 271 (1984); L.C. McDermott and P.S. Schaffer, Research as a guide for curriculum development: An example from introductory electricity. Part I: Investigation of student understanding, Am. J. Phys. 60, 994 (1992); P. S. Schaffer and L.C. McDermott, Research as a guide for curriculum development: An example from introductory electricity. Part II: Design of instructional strategies, ibid. 60, 1003 (1992).

[7] J. Ramadas, Use of ray diagrams in optics, School Sci. 20, 10 (1982); F. M. Goldberg and L. C. McDermott, Student difficulties in understanding image formation by a plane 
mirror, Phys. Teach. 24, 472 (1986); An investigation of student understanding of the real image formed by a converging lens or concave mirror, Am. J. Phys. 55, 108 (1987); P. Colin and L. Viennot, Using two models in optics: Students' difficulties and suggestions for teaching, ibid. 69, S36 (2001).

[8] P. F. Keig and P. A. Rubba, Translation of representations of the structure of matter and its relationship to reasoning, gender, spatial reasoning, and specific prior knowledge, J. Res. Sci. Teach. 30, 883 (1993); W. L. Yarroch, Student understanding of chemical equation balancing, ibid. 22, 449 (1985).

[9] S. Lem, P. Onghena, L. Verschaffel, and W. Van Dooren, On the misinterpretation of histograms and box plots, Educ. Psychol., doi: 10.1080/01443410.2012.674006.

[10] I. Elia, A. Panaoura, A. Gagatsis, K. Gravvani, and P. Spyrou, An empirical four-dimensional model for the understanding of function, in Proceedings of the 30th Conference of the International Group for the Psychology of Mathematics Education, edited by $\mathrm{J}$. Novotná, H. Moraová, M. Krátká, and N. Stehliková (PME, Prague, 2006), Vol. 1, p. 352.

[11] S. Ainsworth, P. Bibby, and D. Wood, Examining the effects of different multiple representational systems in learning primary mathematics, J. Learn. Sci. 11, 25 (2002).

[12] A. Sierpinska, On understanding the notion of function, in The Concept of Function: Aspects of Epistemology and Pedagogy, edited by E. Dubinsky and G. Harel (The Mathematical Association of America, Washington, DC, 1992).

[13] M. Yerushalmy, Student perceptions of aspects of algebraic function using multiple representation software, J. Comput. Assist. Learn. 7, 42 (1991).

[14] A. Van Heuvelen, Learning to think like a physicist: A review of research based instructional strategies, Am. J. Phys. 59, 891 (1991); A. Van Heuvelen and X. Zou, Multiple representations of work-energy processes, ibid. 69, 184 (2001); R. J. Dufresne, W. R. Gerace, and W. J. Leonard, Solving physics problems with multiple representations, Phys. Teach. 35, 270 (1997).

[15] R. Mayer, The promise of multimedia learning: Using the same instructional design methods across different media, Learn. Instr. 13, 125 (2003).

[16] W. Schnotz and M. Bannert, Construction and interference in learning from multiple representation, Learn. Instr. 13, 141 (2003).

[17] R. B. Kozma and J. Russell, Multimedia and understanding: Expert and novice responses to different representations of chemical phenomena, J. Res. Sci. Teach. 34, 949 (1997).

[18] C. J. De Leone and E. Gire, Is instructional emphasis on the use of nonmathematical representations worth the effort?, AIP Conf. Proc. 818, 45 (2006).

[19] D. Rosengrant, A. Van Heuvelen, and E. Etkina, Freebody diagrams: Necessary or sufficient?, AIP Conf. Proc. 790, 177 (2005); D. Rosengrant, E. Etkina, and A. Van Heuvelen, An overview of recent research on multiple representations, ibid. 883, 149 (2007); D. Rosengrant, A. Van Heuvelen, and E. Etkina, Do students use and understand free-body diagrams?, Phys. Rev. ST Phys. Educ. Res. 5, 010108 (2009).
[20] Y. Uesaka and E. Manalo, Active comparison as a means of promoting the development of abstract conditional knowledge and appropriate choice of diagrams in math word problem solving, in Diagrammatic Representation and Inference, Lecture Notes in Artificial Intelligence Vol. 4045, edited by D. Barker-Plummer, R. Cox, and N. Swoboda (Springer-Verlag, Heidelberg, 2006), pp. 181-195.

[21] A. Acevedo Nistal, W. Van Dooren, G. Clarebout, J. Elen, and L. Verschaffel, Representational flexibility in linearfunction problems: A choice/no-choice study, in Use of Representations in Reasoning and Problem Solving: Analysis and Improvement, edited by L. Verschaffel, E. de Corte, T. de Jong, and J. Elen (Routledge, Oxon, 2010).

[22] A. Acevedo Nistal, W. Van Dooren, and L. Verschaffel, Students' reported justifications for their representational choices in linear function problems: An interview study, Educ. Stud., doi: 10.1080/03055698.2012.674636.

[23] A. diSessa, D. Hammer, B. Sherin, and T. Kolpakowski, Inventing graphing: Meta-representational expertise in children, J. Math. Behav. 10, 117 (1991).

[24] A. diSessa and B. Sherin, Meta-representation: An introduction, J. Math. Behav. 19, 385 (2000).

[25] A. diSessa, Students' criteria for representational adequacy, in Symbolizing, Modeling and Tool Use in Mathematics Education, edited by K. Gravemeijer, R. Lehrer, B. van Oers, and L. Verschaffel (Kluwer Academic, Dordrecht, 2003).

[26] P. B. Kohl and N. D. Finkelstein, Student representational competence and self-assessment when solving physics problems, Phys. Rev. ST Phys. Educ. Res. 1, 010104 (2005).

[27] K. R. Koedinger and M. J. Nathan, The real story behind story problems: Effects of representations on quantitative reasoning, J. Learn. Sci. 13, 129 (2004).

[28] D.E. Meltzer, Relation between students' problemsolving performance and representational format, Am. J. Phys. 73, 463 (2005).

[29] E. Mazur, Peer Instruction: A User's Manual (PrenticeHall, Upper Saddle River, NJ, 1996).

[30] D. De Bock, W. Van Dooren, D. Janssens, and L. Verschaffel, Improper use of linear reasoning: An in-depth study of the nature and the irresistibility of secondary school students' errors, Educ. Stud. Math. 50, 311 (2002); D. De Bock, L. Verschaffel, and D. Janssens, The effects of different problem presentations and formulations on the illusion of linearity in secondary school students, Math. Think. Learn. 4, 65 (2002); D. De Bock, W. Van Dooren, L. Verschaffel, and D. Janssens, Secondary school pupils' improper proportional reasoning: An in-depth study of the nature and persistence of pupils' errors, in Proceedings of the 25th Conference of the International Group for the Psychology of Mathematics Education, Utrecht, The Netherlands, 2001, edited by M. van den Heuvel-Panhuizen, Vol. 2, pp. 313-320 (unpublished); D. De Bock, L. Verschaffel, and D. Janssens, The predominance of the linear model in secondary school students' solutions of word problems involving length and area of similar plane figures, Educ. Stud. Math. 35, 65 (1998). 
[31] D. De Bock, W. Van Dooren, and L. Verschaffel, Students' overuse of linearity: An exploration in physics, Res. Sci. Educ. 41, 389 (2011).

[32] P. B. Kohl and N. D. Finkelstein, Effects of representation on students solving physics problems: A fine-grained characterization, Phys. Rev. ST Phys. Educ. Res. 2, 010106 (2006).

[33] L. N. Walsh, R. G. Howard, and B. Bowe, Phenomenographic study of students' problem solving approaches in physics, Phys. Rev. ST Phys. Educ. Res. 3, 020108 (2007).
[34] H. A. Simon and J. R. Hayes, The understanding process: Problem isomorphs, Cogn. Psychol. 8, 165 (1976).

[35] C. Singh, Assessing student expertise in introductory physics with isomorphic problems. II. Effect of some potential factors on problem solving and transfer, Phys. Rev. ST Phys. Educ. Res. 4, 010105 (2008).

[36] L. Verschaffel, J. Torbeyns, B. De Smedt, K. Luwel, and W. Van Dooren, Strategy flexibility in children with low achievement in mathematics, Educ. Child Psychol. 24, 16 (2007). 